\title{
THE STRUCTURE OF NON-ARGUESIAN LATTICES
}

\author{
BY ALAN DAY AND BJARNI JÓNSSON
}

\begin{abstract}
The purpose of this research is to answer the question: How does a modular lattice fail to be Arguesian?
\end{abstract}

The Arguesian law was introduced in [6] to provide a lattice identity, stronger than Dedekind's modular law, that holds in lattices of submodules, of normal subgroups, and more generally, of permuting equivalent relations. It was so named because a projective plane satisfies Desargues' Axiom just in case its (modular) lattice of subspaces satisfies the Arguesian identity.

In [2 and 8] this identity was shown to be equivalent to an implication that naturally reflects Desargues' geometrical statement. A triangle in $L$ is an ordered triple $\mathbf{x}=\left\langle x_{0}, x_{1}, x_{2}\right\rangle$ in $L^{3}$. Given two triangles, $\mathbf{a}$ and $\mathbf{b}$, in $L$, we define $p:=p(\mathbf{a}, \mathbf{b})=\prod\left(a_{i}+b_{i}\right.$ : all $\left.i\right)$ and $c_{i}:=c_{i}(\mathbf{a}, \mathbf{b})=\left(a_{j}+a_{k}\right)\left(b_{j}+b_{k}\right)$ for $\{i, j, k\}=\mathbf{3}$. $\mathbf{a}$ and $\mathbf{b}$ are called centrally perspective if $\left(a_{0}+b_{0}\right)\left(a_{1}+b_{1}\right) \leq$ $a_{2}+b_{2}$, and axially perspective if $c_{2} \leq c_{0}+c_{1}$. A lattice is called Arguesian if every pair of centrally perspective triangles is axially perspective.

The above definition still lacks certain geometric properties: It is not $\{0,1,2\}$-symmetric; and the "vertices" of the triangles can be arbitrary elements of $L$. To obtain a more pungent geometric flavour, a quadruple $\mathbf{x}=\left\langle x_{0}, \ldots, x_{3}\right\rangle$ in $L$ is said to be in general position over $u$ in $L$ if, for any 3 distinct indices, $x_{i}\left(x_{j}+x_{k}\right)=u$. Note that $u$ is necessarily the meet of all the $x_{i}$ 's. A perspectivity configuration (or $\mathrm{PC}$ ) in $L$ is a pair of triangles, $\langle\mathbf{a}, \mathbf{b}\rangle$, in $L$ satisfying: (1) $a_{i}+p=b_{i}+p=a_{i}+b_{i}$, all $i$; and (2) $\langle\mathbf{a}, p\rangle$ and $\langle\mathbf{b}, \boldsymbol{p}\rangle$ are quadruples in general position. A PC in $L$ is called Arguesian if its triangles are axially perspective. The results of $[\mathbf{1}]$ can be reformulated.

LEMMA 1. Let $\langle\mathbf{a}, \mathbf{b}\rangle$ be a PC in L. Then

(1) $\mathbf{a}$ and $\mathbf{b}$ are triply centrally perspective [i.e. $\left.p=\left(a_{i}+b_{i}\right)\left(a_{j}+b_{j}\right), i \neq j\right]$;

(2) if $\langle\mathbf{a}, \mathbf{b}\rangle$ is Arguesian, then $c_{0}+c_{1}=c_{0}+c_{2}=c_{1}+c_{2}$.

THEOREM 1. A modular lattice $L$ is Arguesian if and only if every perspectivity configuration in $L$ is Arguesian.

Although non-Arguesian projective planes provide the archetypical examples of modular non-Arguesian lattices, there are other ways to construct such models. In [7] the Hall-Dilworth gluing [3] was applied to two Arguesian planes of the same order to produce a modular lattice that is Arguesian just in case the gluing is conservative: quadrangular sextuples of lines are glued to quadrangular sextuples of points. Using Herrmann's $S$-Verklebte Summen [4], these authors, Herrmann [5], and Pickering [9] have produced more intricate

Received by the editors January 17, 1985.

1980 Mathematics Subject Classification. Primary 06C05; Secondary 51A05. 
gluings of Arguesian planes. These gluings provide us with further examples. Our principal result shows that the geometric flavour of these examples is no accident.

The following observation will bring out more clearly the analogy between our result and the classical Arguesian configuration of 10 points and 10 lines. Let $S^{[n]}$ be the set of all $n$-element subsets of $S$. A projective plane is nonArguesian iff there exist distinct points $p_{i}, i \in \mathbf{5}^{[2]}$ and distinct lines $l_{j}, j \in$ $5^{[3]}$ such that, with precisely one exception, if $i \subseteq j$, then $p_{i}$ lies on $l_{j}$.

THEOREM 2. A modular lattice $L$ is non-Arguesian if and only if there exist, in the ideal lattice of $L$, intervals $P_{i}=u_{i} / z_{i}$ and $L_{j}=u_{j} / z_{j}, i \in \mathbf{5}^{[2]}$ and $j \in \mathbf{5}^{[3]}$, that are nondegenerate projective planes, points $p_{i} \in P_{i}$ and lines $l_{j} \in L_{j}$ subject to the following conditions:

(1) $u_{i}=u_{j} u_{j^{\prime}}$, if $j \cap j^{\prime}=i$;

(2) $z_{j}=z_{i}+z_{i^{\prime}}$, if $j=i \cup i^{\prime}$

(3) If $i \neq\{3,4\}$, then the three elements of the form $u_{i} l_{j}, i \subseteq j$, are distinct lines in $P_{i}$ through $p_{i}$;

(4) If $j \neq\{2,3,4\}$, then the three elements of the form $z_{j}+p_{i}, i \subseteq j$, are distinct points in $L_{j}$ through $l_{j}$;

(5) For $i=\{3,4\}$, the three elements $u_{i} l_{j}$ are nonconcurrent lines in $P_{i}$;

(6) For $j=\{2,3,4\}$, the three elements $z_{j}+p_{i}$ are noncollinear points in $l_{j}$.

OUTLINE OF PROOF. If $L$ is non-Arguesian, then $L$ contains a nonArguesian perspectivity configuration, $\langle\mathbf{a}, \mathbf{b}\rangle$.

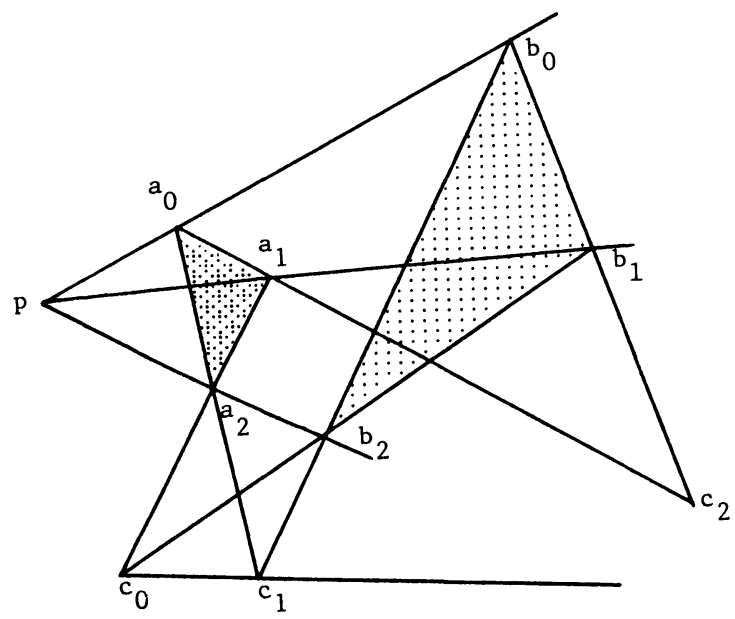


A detailed study of this PC produces other "equivalent" PC's, e.g. $\left\langle\left\langle c_{1}, c_{0}, a_{2}\right\rangle,\left\langle b_{0}, b_{1}, p\right\rangle\right\rangle$ and $\left\langle\left\langle c_{1}, b_{2}, a_{2}\right\rangle,\left\langle\left(a_{0}+a_{1}\right)\left(c_{0}+c_{1}\right), b_{1}, a_{1}\right\rangle\right\rangle$, thus showing that the positioning of the 10 points is irrelevant. Projectivities between certain intervals show that suitably defined "sub-PC's" are determined uniquely by any co-ordinate. This allows us to pass to the ideal lattice and, via a maximality argument, produce a non-Arguesian $\mathrm{PC}$, also called $\langle\mathbf{a}, \mathbf{b}\rangle$, with $c_{2}$ covering $c_{2}\left(c_{0}+c_{1}\right)$. This covering relation induces the 20 projective planes, two of which are

(1) $P_{\{3,4\}}: u_{\{3,4\}}:=c_{2}+\left[a_{0}+a_{1}\right]\left[c_{0}+c_{1}\right]+\left[b_{0}+b_{1}\right]\left[c_{0}+c_{1}\right]$, and $z_{\{3,4\}}:=$ $c_{2}\left[c_{0}+c_{1}\right]$

(2) $L_{\{2,3,4\}}: u_{\{2,3,4\}}:=c_{0}+c_{1}+c_{2}$, and $z_{\{2,3,4\}}:=\prod\left(c_{i}+c_{j}:\right.$ all $\left.i \neq j\right)$.

\section{REFERENCES}

1. A. Day, A note on Arguesian lattices, Arch. Math. 3, Scripta Fac. Sci. Nat. Ujep Brunensis XIX (1983), 117-124.

2. G. Grätzer, B. Jónsson and H. Lakser, The amalgamation property in equational classes of modular lattices, Pacific J. Math. 45 (1973), 507-524.

3. M. Hall, Jr. and R. Dilworth, The embedding theorem for modular lattices, Ann. of Math. (2) 45 (1944), 450-456.

4. Ch. Herrmann, S-Verdebte Summen von Verbanden, Math. Z. 130 (1973), 255-274.

5. __ On the word problem for modular lattices with four generators, Math. Ann. 265 (1983), 513-527.

6. B. Jónsson, Modular lattices and Desargues' theorem, Math. Scand. 2 (1954), 295-314.

7. __ Arguesian lattices of dimension $n \leq 4$, Math. Scand. 7 (1959), 133-145.

8. B. Jónsson and G. Monk, Representation of primary Arguesian lattices, Pacific J. Math. 30 (1969), 95-139.

9. D. Pickering, On minimal non-Arguesian lattice varieties, $\mathrm{PhD}$ Thesis, Univ. of Hawaii, 1984.

Department of Mathematical Sciences, LAKehead University, ThunDER BAY, ONTARIO, CANADA P7B 5E1

Department of Mathematics, VANDERBILt University, NASHVille, TENNESSEE 37235 